\title{
Evaluation of Environmental Impact Assessment Utilization: The Case of United Kingdom Construction Projects
}

\author{
Benjamin Edokpolo ${ }^{1}$ and Tarila Zuofa ${ }^{2}$
}

\begin{abstract}
The environmental impact of construction activities have remained important issue as a result, most construction industry stakeholders are facing the huge challenge to achieve a more resource-efficient and environment friendly society. This study critically evaluates the process of managing the implementation of mitigating strategies aimed at reducing the negative environmental impacts associated with the construction of a new 180MVA 275/66kV transformer for the National Grid Substation in West Boldon, Tyne and Wear, United Kingdom. 20 copies of questionnaires were distributed to members of the project team to investigate the implementation of the mitigating proposed measures in the environmental impact assessment. The survey was undertaken during the project execution to identify procedures for reducing or eliminating negative impacts and to also suggest ways of improving the implementing of the environmental impact assessment process. The questionnaires which had a response rate of $75 \%$ were collated and plotted as bar graphs and pie charts for further evaluation. The main findings indicated that majority of the participants (90\%) identified the use of standard assessment procedures and tools such as environmental impact assessment, environmental checklists and the environmental management plan as well as environmental training as strategies for increasing the awareness for project team members on environmental concerns. The result also suggests that environmental training should be an ongoing process for all personnel on site during the construction of the project in sustaining the awareness, knowledge, skills and attitude.
\end{abstract}

Keywords: construction projects, environmental impact assessment, evaluation, project team, United Kingdom.

\section{Introduction}

In our present world, we are faced with the challenges of access to portable water, polluted air, contaminated land and global warming as a result of human activities on natural environment. Adequate consideration should be given to the environment when carrying out activities that will have negative impacts on the environment (Noble, 2010). A project can be referred to as a process that has a clearly defined start date and finish date, a set of tasks, and a budget developed to solve a well-defined goal or objective (Collyer et al. 2010). The end point of a project or objective needs to be well articulated in the early stages of scheduling and monitoring the activities of the project for its successful completion (Zuofa and Ochieng, 2011).

\footnotetext{
1 Griffith School of Engineering, Griffith University, Nathan Campus, Brisbane, 4111, QLD, Australia, Tel: +61 (0)7 373573619, Email: b.edokpolo@griffith.edu.au

2 University of Westminster 35 Marylebone Road, London, NW1 5LS. Email: t.zoufa@westminster.ac.uk
} 
A construction project consists of different processes in the assembling or building of infrastructures such as the construction of buildings, roads, bridges and industries (Artto et al. 2008). Thomas et al. (2008), suggested that the successful execution of construction projects requires a project manager, design and construction engineers as well as a good project team. The process in constructing a project involves selecting a project site, planning the project, designing the project to suit the environment and implementing the project on the chosen site (Kerzner, 2009). From the environmental point of view developmental project will not only have positive impact in terms of improving the lives of people, but also negative impacts that are associated with the implementation of the project such as land, air and water pollution as well as threats to endangered species (Toro et al. 2010).

Environment impact assessment (EIA) is a set of procedures aimed at contributing to development as well as serving as a veritable tool for management in order to minimise environmentally negative effects of the project when in the construction and operational phases (Ali - Damkhi et al. 2008). (Elliott and Thomas, 2009), view environment impact assessment as a tool used in estimating the likely and most detrimental impacts that a project might have on its surrounding environment. Hence, environmental impact assessment serves as a tool used in estimating the likely and most detrimental impacts that a project might have on its surrounding environment. Also, measures in mitigating the identified negative environmental impacts should be carried out prior to a project to incorporate in the planning and design stages of the project (Toro et al. 2013).

The aim of this study was to focus on the effective implementation of the environmental management plan proposed in the environmental impact assessment report in minimising as well as completely removing the impacts.

\section{Methodology}

\section{Description of the Construction Project}

The national grid station is the owner and operator of West Boldon substation generator and supplier of energy to customers that cut across United Kingdom, the substation is located in West Boldon Tyne and Wear, the project is supplying a new transformer (180MVA $275 / 66 \mathrm{kV}$ ) to the substation. The construction project was ongoing and the main construction activities were;

- The refurbishment of old office buildings

- Erection of new facilities

- Demolition of unwanted facilities

- Civil works

\section{Data Collection}

The use of questionnaire was used for the study in obtaining the relevant information that will be useful in evaluating the measures for mitigating the environmental impact that are predicted in the environmental impact assessment report. The use of questionnaire is a common and useful tool in acquiring facts that are qualitative and quantitative in management research (Denscombe, 2014). Also, to maximize the use of questionnaire is by asking the right questions to easily assess the relationships that exists between variables to enhance the analysis of the findings (Yin, 2013).

Therefore to evaluate the mitigating measures during the implementation of the environmental impact assessment report for the project, the questions was not many and written in a clear language that was easily understood by all and not time consuming. 


\section{Medium for Distributing Questionnaire}

There were several visits to the construction site to discuss with the site manager on the involvement of the project team in the study. The questionnaires were discussed and approved by the site manager. The participating project team involved in the process was advised to give their opinion on areas of concern reflected in the questionnaire. Finally the questionnaires were collected by the site manager and distributed to members of the project team.

\section{Statistical Analysis}

The data for analysis was obtained from the response to the questionnaire by members of the project team and was analysed with the use of excel statistical software in displaying the findings in a bar chart and pie chart and also explaining the data that cannot be represented with simply arithmetic percentage and ratios for the analysis.

\section{Results and Discussion Background}

This study shows the response to the questionnaire on managing the implementation of environmental impact assessment in the construction of project. The impacts were identified during scoping of the environmental impact assessment process while the measures for mitigating and eliminating the identified impacts were proposed in the environmental management plan which is in line with the aim of this study; investigation of the process of implementing the measures of impacts mitigation in ongoing construction activities in project site. In achieving the aim of the study, questions on the various ways of implementing the measures were asked to members of the project team, and the response to these questions are analyzed in the various sub headings below.

\section{Number of Participants}

Twenty sample questionnaires were given to the project team on site, fifteen of the twenty members of the project team responded to the questionnaire by giving their individual opinion to the questionnaire thus, $75 \%$ of the project team participated in the survey. The three managers within all gave a very positive response to the questions due to their level of experience however, their results were not separated from the twelve members of the project team that took part in the survey as a result of the small size of the sample survey.

\section{Graphical Representation and Evaluation of Project Team Response to Questionnaire}

The subsequent analysis of the findings of the results is presented in bar graphs and pie chart in getting a better representation of the response of the project team on the level of importance of the various procedures and tools in managing the implementation of mitigating measures in eliminating or reducing the possible negative impacts relating to the construction of the project on the environment. The high, medium, low and not important scale is used in the questionnaire (Figure 1) was to evaluate the response of the project team on the procedure and tool that is most useful to the project team during the construction of the project bearing in mind that these are all important tools of eliminating or minimizing negative impacts on the environment in the construction of the project.

As shown in Figure 1, the project team is very much aware of the environmental issues that surround the project prior to the commencement of construction activities, environmental training was given to all personnel on site on the possible negative impacts 
that are associated with the project identified in the environmental impact assessment for the project, the likely factors that can affect the environment from the use of resources (human, technology and natural resources), the impacts of waste that is generated from the project on the environment if not properly disposed, and their responsibilities in implementing the environmental management plan that is in place to control and regulate the construction activities in mitigating the identified negative impacts and avoiding new impacts that may occur during the construction of the project.

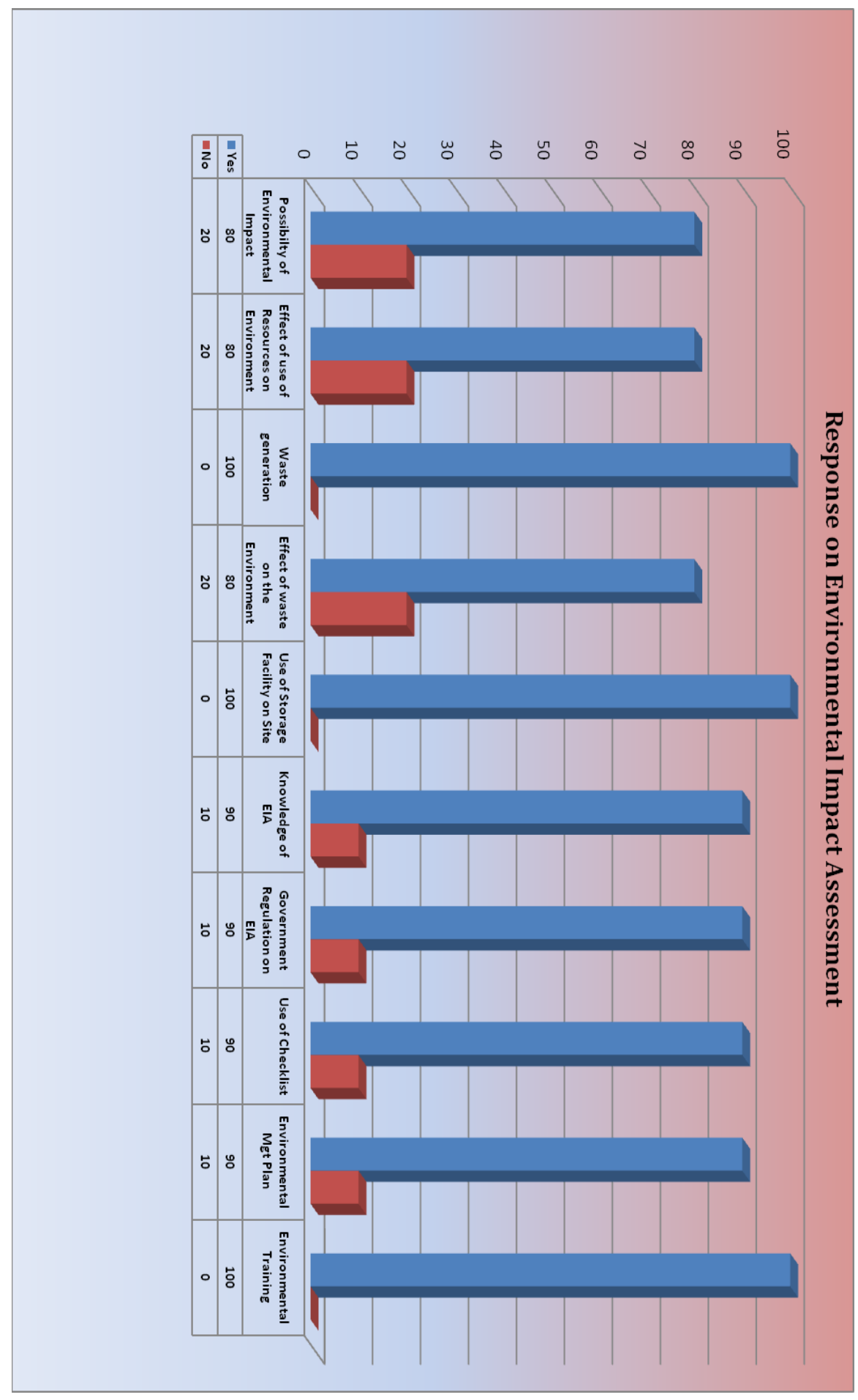

Figure 1. Summary of the Response of the Project Team to the Questionnaire Survey 
Hence there is a high response of YES as against NO to the question. Although quite a number of personnel have not been involved in a similar project but, there is general awareness that the project is likely to have an impact that will be consequential on the environment, and the use of technology, energy, diesel, raw material, water and human activities can affect the environment as well as the wastes that will be generated in the construction pf the project, and they are also aware that environmental impact assessment was carried out as part of a project not because the sponsor wish to do it but, because it is a governmental regulation that for a project that is likely to have a negative impact on the environment (Surrey County Council, 2009), going by this, environmental impact assessment was carried out and putting in place an environmental management plan in controlling and monitoring of the impacts.

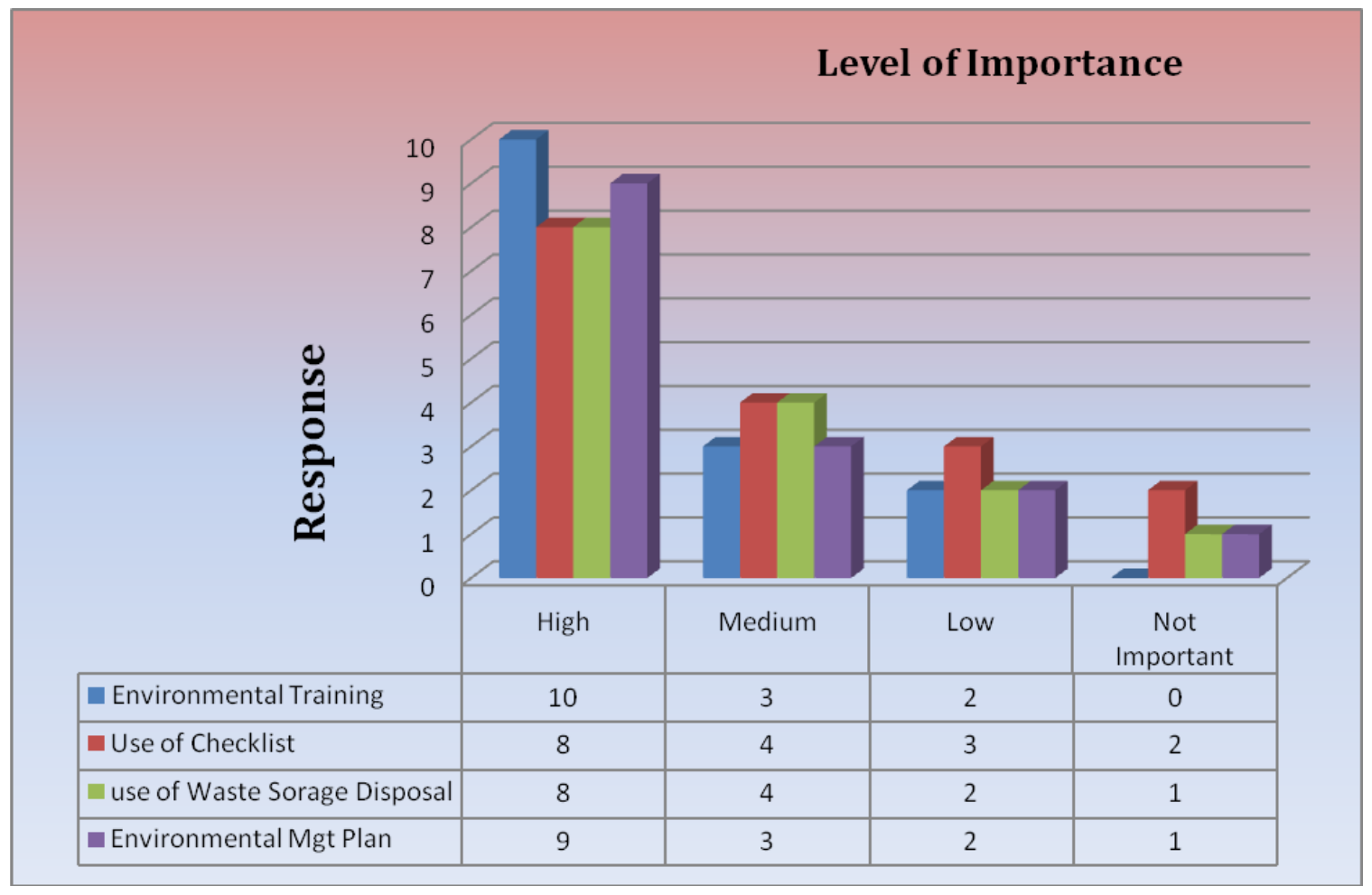

Figure 2. Level of Importance of standard assessment procedures and environmental tools

Figure 2 represents the level of importance of the standard procedures and tools in managing the implementation of mitigating measures in eliminating or reducing the possible negative impacts relating to the construction of the project on the environment. As shown in Figure 2, the high, medium, low and not important scales were used in evaluating the response of the project team. Environmental training for the project team is highly rated in terms of its importance to the project, followed by environmental management plan and the use of checklist and waste storage facility on site were equally rated. As suggested by Dwivedula and Bredillet (2009), training for project teams do not only provide the required skills, knowledge, altitude in solving problems, it also creates awareness amongst the project team on the issues that affects the implementation of the project. The environmental impact assessment and environmental management plan serves as a tool not only in the identification of these impacts but also measures in mitigating the impacts (Wathern, 2013). Hence, if the project team is not given an environmental training on these areas of the project it is unlikely that the measures be well implemented. However, the result above shows that 
even if environmental training got the highest rating, the use of checklist, waste storage on site and environmental management plan are all very important in reducing and if possible the complete removal of the impacts that is associated with the construction project.

The evaluation of project team response on the effectiveness of environmental training for the project team before, during and after the construction of the project is shown in Figure 3.

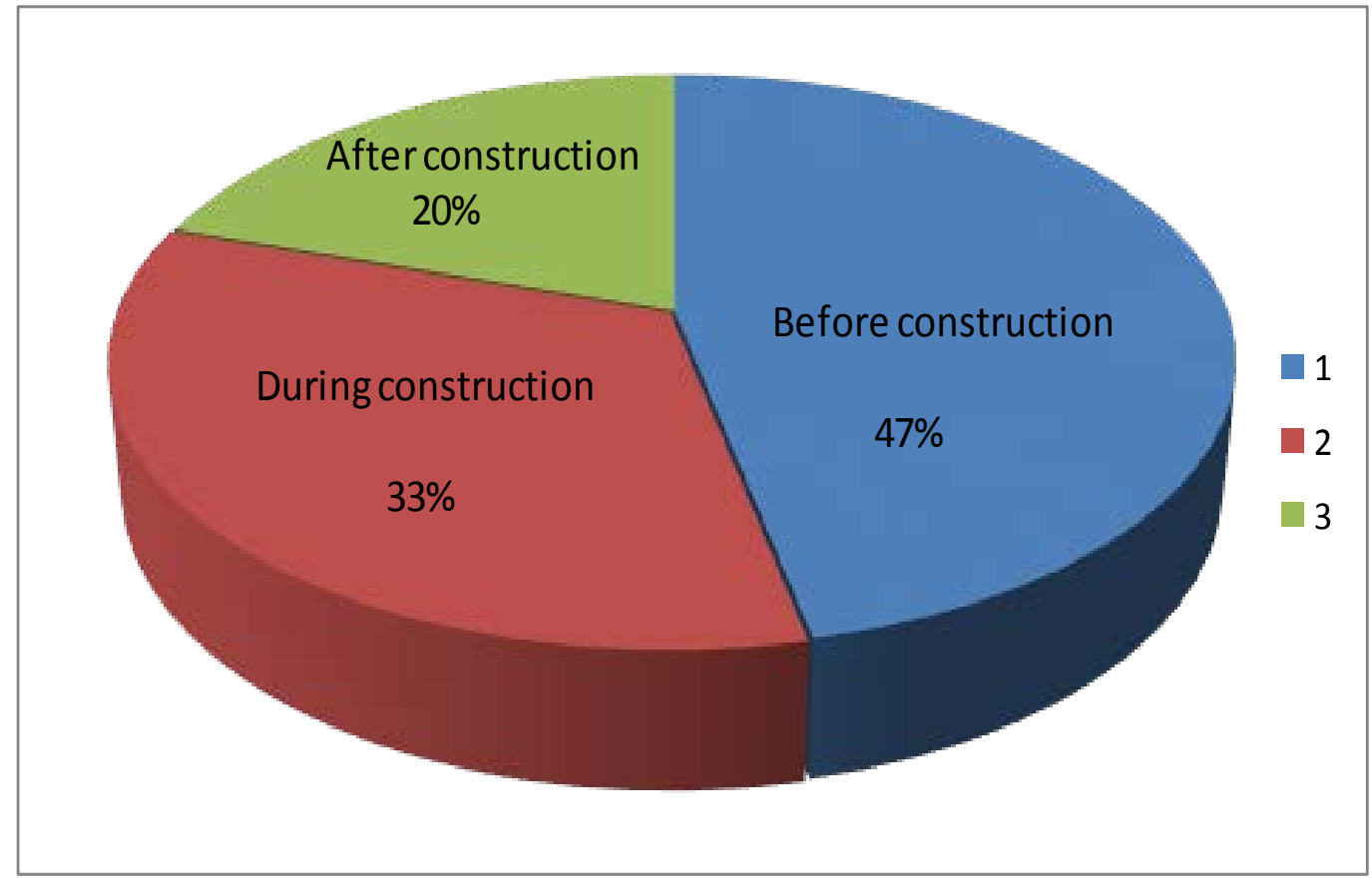

Figure 3. Effectiveness of environmental training in the life cycle of a project

From the result, $47 \%$ of the project team supports the importance of having environmental training before the start of construction activities, while 33\% consider it to be more useful during construction stage and the remaining $20 \%$ of the project team are of the opinion it should be done after the project. Prior to the construction of the project, environmental training was given to the project team in creating an awareness on environmental issues and concerns relating to the construction of the project. The training can also be useful during the construction of the project in assessing the response of the project team in dealing with the environmental issues and concerns surrounding the project. Also, providing training for the project team at the end of the project was regarded to be useful in evaluating the impact of the environmental training during the project and the lessons learnt in the construction of the project. This was considered helpful in creating the awareness and experience in dealing with environmental concerns and issues in the construction of similar projects in the future. As shown in the analysis of the questionnaire survey, most of the members of the project team have been involved in a similar construction project in the past and based on their previous experience in the construction of a similar project, most members of the project team expects the construction of the project to have negative impacts on the environment. Going by the research studies of Ochieng et al. (2013) developmental projects mainly construction projects do have both positive and negative impacts on the environment therefore, in as much as the project is going to improve the environment positively, there are negative impacts associated with the project that are likely to affect the environment. 
From the evaluation of the empirical study and the review of literatures relevant to this study, in enhancing environmental sustainability it is necessary for the environment to retain its state before the construction even after the construction of the project (Morelli, 2013). In the construction of the project, there were likely negative impacts on the environment identified in the environmental impact assessment report prior to the implementation of the construction project thereby putting in place mitigating measures for eliminating the impacts that are likely to affect the environment. To successfully manage the mitigating measures of eliminating the impacts, some procedures and tools amongst others were utilized by the management and project team such as the regular use of environmental checklist, environmental management plan, training for the project team in creating the required knowledge, skills and understanding of the measures put in place to mitigate the negative impacts that will affect the environment (Senarente and Sexton, 2009). However, there could still be other useful tools that can be utilized in managing the implementation of the mitigating measures in reducing or completely removing the impacts relating to the construction of the project not considered in the research study and also the possibility of errors in the findings of the research studies considering that this result was based on the opinion of members of the project team in their response to the questionnaire survey as well as the literatures reviewed. Hence the findings of this study can still be subject to further research in evaluating the process of managing the implementation of the mitigating measures of likely negative impacts on the environment relating to the construction of a project through the utilization of environmental assessment.

\section{Conclusions}

The evaluation of managing the implementation of mitigating measures as a result of negative impacts on the environment identified in the EIA report in the construction of office buildings and the supply of new transformer (180MVA 275/66kV) national grid substation in West Boldon was carried out using questionnaires. 15 members of the project team that includes 3 site managers participated in the study. The findings in this research study shows that EIA is a useful tool in the identification, evaluation and the analysis of possible negative impacts that will affect the environment. In addition, EIA also proposes measures that will minimize and if possible eliminate the likely impacts in the construction of a project. More than $90 \%$ of the project team were in support of applying useful tools such as environmental impact assessment, environmental checklist, environmental management plan, ways and means of waste disposal and environmental training are very useful tools for managing environment impacts during the construction project. However, the evaluation of these procedures and tools in minimizing and eliminating the negative impacts associated with the construction of the project suggests they have been properly utilized by the project team.

\section{References}

Ali- Damkhi, A. M., Abdul- Wahab, S. A., Atkhalaf, N. and Al-Nafisi, A. S., 2008. Developing environmental impact statement (EIS) guidelines for the management of environmental quality at major projects in Kuwait: Management of Environmental Quality. An International Journal. 19 (6), 670-689.

Artto, K., Martinsuo, M., Dictrich, P. and Kujala, J., 2008. Project strategy types and their contents in innovation project. International Journal of Project Management. 1 (1), 49-70.

Collyer, S., Warren, C., Hemsley, B., and Stevens, C., 2010. Aim, Fire, Aim-Project

Planning Styles in Dynamic Environments. Project Management Journal, 41(4), 108-121. 
Denscombe, M., 2014. The good research guide: for small-scale social research projects. McGraw-Hill Education (UK).

Dwivedula, R., and Bredillet, C. N., 2010. The Relationship between Organizational and Professional Commitment in the Case of Project Workers: Implications for Project

Management. Project Management Journal, 41(4), 79-88.

Elliott, M., Thomas, I., 2009. Environmental Impact Assessment in Australia: Theory and Practice (Third ed.) Federation Press, Australia.

Kerzner, H., 2009. Project Management: A Systems Approach to Planning, Scheduling, and Controlling, 10 Edition, John Wiley \& Sons Inc, New Jersey, USA.

Morelli, J. (2013). Environmental sustainability: A definition for environmental professionals. Journal of Environmental Sustainability, 1(1), 2.

Noble, B. F., 2010. Introduction to Environmental Impact Assessment: A Guide to Principles and Practice Oxford University Press, Oxford, United Kingdom.

Ochieng, E., Price, A., and Moore, D., 2013. Management of Global Construction Projects. Palgrave Macmillan.

Surrey County Council 2009. Environmental impact assessment (EIA) guidance note 2. A Guide to Schedule of the Town \& County Planning (environmental Impact Assessment) (England \& Wales) Regulations 1999.

Senarente, S. and Sexton, M. G., 2009. Role of knowledge in managing construction project change. Engineering, Construction and Architectural Management. 16 (2), 186-200.

Thomas, M., Jacques, P. H., Adams, J. R., and Kihneman-Wooten, J., 2008. Developing an Effective Project: Planning and Team Building Combined. Project Management Journal, 39(4), 105-113.

Toro, J., Requena, I., Duarte, O. and Zamorano, M. A., 2013. Qualitative method proposal to improve environmental impact assessment. Environ. Impact Assess. Rev., 43, 9-20

Toro, J., Requena, I., Zamorano, M., 2010. Environmental impact assessment in Colombia: critical analysis and proposals for improvement. Environ. Impact Assess. Rev., 30, 247-261.

Wathern, P., 2013. Environmental impact assessment: theory and practice. Routledge.

Yin, R. K., 2013. Case study research: Design and methods. Sage publications.

Zuofa, T., and Ochieng, E. G., 2011. Project managers perception of risk factors in heavy engineering construction projects: Case of offshore projects In: C. Egbu and E.C.W. Lou, eds. Proceedings $27^{\text {th }}$ Annual ARCOM Conference, 5-7 September 2011, Bristol, UK. Association of Researchers in Construction Management, 985-993. 